\title{
VARIACIONES NEOLIBERALES EN LA REPRODUCCIÓN DE LOS CUERPOS: MERCADO, TRABAJO Y CUIDADOS*
}

\author{
Neoliberal Shifts on the Reproduction of Bodies: Market, Work, and Care
}

Iker Jauregui Giráldez

\author{
Universidad Complutense de Madrid \\ ikerjaur@ucm.es
}

\begin{abstract}
Resumen:
El objetivo del presente artículo es analizar las políticas de reproducción de los cuerpos en el neoliberalismo desde una perspectiva laboral amplia (dentro y fuera de la relación asalariada). Para ello se traza, en primer lugar, una genealogía rápida de las normas reproductivas desde el fordismo hasta la entrada en crisis del sistema welfarista, teniendo en cuenta las diferentes configuraciones del salario, del consumo y del trabajo doméstico no pagado. A continuación, se analiza el neoliberalismo en tanto que proyecto político -materializado en medidas laborales concretas- y corpus discursivo -la ideología y discursos teóricos que lo configuran- como un proceso de privatización de la reproducción a varios niveles.
\end{abstract}

Palabras clave:

Reproducción, cuerpo, trabajo, neoliberalismo, privatización.

\begin{abstract}
:
The main purpose of this paper is to study the reproduction of bodies under neoliberalism from a wide labour perspective (inside and outside the wage relation). First, we draw a brief genealogy of the different reproductive norms from fordism to welfare states' crisis (taking account of wage, consumption and unpaid housework configurations). Then, neoliberalism is analyzed in its diverse labor policies and in its ideological and theoretical discourses as a privatization process of reproduction at various levels.
\end{abstract}

Keywords:

Reproduction, Body, Work, Neoliberalism, Privatization.

Recibido: 22/10/2018

Aceptado: 15/12/2018

\footnotetext{
* Esta investigación ha sido posible gracias a una beca FPU del Ministerio de Educación, Cultura y Deporte y se enmarca en el Proyecto de Investigación «Naturaleza Humana y Comunidad IV» (FFI2017-83155-P).
} 
Es ya habitual presentar al capitalismo con metáforas víricas; pensarlo en su lógica expansiva como un contagio impredecible y continuo que absorbe espacios en apariencia extraeconómicos. En ocasiones es el propio capitalismo, y no sus críticos, quien se regala a sí mismo este tipo de juegos expresivos y los materializa: pensemos en la realidad colonial, auténtica absorción de espacios marginados de las lógicas mercantiles. Ya sea el territorio, la cultura, lo político o lo comunitario, está en el ADN del capitalismo la conquista de lo que en otro tiempo era un límite. El capital, por tanto, transgrede límites. Pero en cada transgresión, en cada salto de frontera, hay, evidentemente, una absorción productiva, un uso deliberado. Es, de nuevo, el caso del territorio, con la expropiación colonial, o el caso de la cultura y lo político, con la intervención constante de la lógica del beneficio empresarial. (Sobra decir que toda transgresión implica la desaparición de lo transgredido: el límite es la marca de las diferencias cualitativas entre lo que hay más acá y lo que hay más allá, y cuando éste se transgrede desaparecen las diferencias y pocos pueden distinguir ya un político o un gestor cultural de un empresario). Uno de esos espacios fronterizos, el cuerpo, refleja de manera transparente ciertos conflictos en esa política de las transgresiones y las absorciones productivas: sin él, sin el cuerpo, el capitalismo -desde sus niveles más simples, desde la producción misma- no es nada (pues el cuerpo, la fuerza de trabajo ${ }^{1}$, es el motor principal de la generación de valor), pero en él puede toparse con límites difíciles de transgredir. El cuerpo, la fuerza de trabajo, se explota, pero es explotable dentro de ciertos límites. Dicho con otras palabras, el capitalismo tiene que «estar pendiente» de los cuerpos. Podría decirse que la historia del capitalismo es la historia de las diferentes formas de pensar y tratar los cuerpos; de las diferentes formas de disciplinar ese límite que es el cuerpo, de explotarlo, de reproducirlo; de las diferentes formas de someterlo a lógicas, tiempos y movimientos que no tienen que ver con los cuerpos.

Por su carácter fronterizo -entre lo explotable y lo que no es ni siempre ni del todo explotable; entre lo absolutamente rentable o económico y lo absolutamente norentable; entre, digamos, su permeabilidad a las lógicas mercantiles y su radical impermeabilidad a las mismas- el cuerpo nos ofrece una óptica imprescindible desde la que analizar el desarrollo del capitalismo (un espacio epistemológico privilegiado, podríamos decir). Una de las vías desde donde analizar las políticas corporales del capitalismo -la que aquí exploraremos- tiene que ver con la reproducción de los cuerpos.

\footnotetext{
${ }^{1}$ Si bien no se trata de dos términos analíticamente intercambiables, para nuestro propósito utilizaremos «cuerpo» como ampliación conceptual de «fuerza de trabajo» por una serie de motivos: en primer lugar de índole político (por un lado, porque «fuerza de trabajo», al menos si se entiende como aquella mercancía que se vende en el mercado laboral capitalista, excluye automáticamente otros cuerpos explotables y explotados en el sistema de reproducción social capitalista; por otro lado porque al hablar también de «cuerpos» se avanza en la comprensión de una agenda política que sitúa al cuerpo, dentro y fuera de la relación laboral, en un lugar central) y de índole filosófico (para así incluir los procesos de reproducción de la fuerza de trabajo en la dinámica general de acción disciplinaria y control de los cuerpos, tal y como sugieren autores como Foucault).
} 


\section{LA REPRODUCCIÓN DE LA FUERZA DE TRABAJO EN MARX (MÁS ALLÁ DE MARX)}

Ya en Marx encontramos, si bien superficialmente, algunas consideraciones acerca de la reproducción de la fuerza de trabajo. "El capitalista -escribe Marx en el capítulo XXI del libro primero de El Capital (2017: 660) - [...] constantemente produce la fuerza de trabajo como fuente subjetiva y abstracta de riqueza, [...] existente en la mera corporeidad del obrero; en una palabra, produce al trabajador como asalariado. Esta constante reproducción o perpetuación del obrero es la [conditio] sine qua non de la producción capitalista". El proceso de producción capitalista presupone, por tanto, el proceso de (re)producción del obrero mismo, la subsistencia material permanente del trabajador (aquello que le permitirá, a ojos del capitalista, presentarse día tras día, semana tras semana, en la fábrica). El salario mismo, dirá Marx, debe entenderse como el pago, en concepto de «alquiler», dirigido a sufragar los gastos reproductivos del trabajador, una "cantidad determinada de músculo, nervio, cerebro, etc. humanos, que es necesario reponer" (2017: 230) ${ }^{2}$. Se trata, por tanto, de una nueva norma reproductiva que vincula el cuerpo del trabajador, su salario, y el consumo de los medios de subsistencia con el que él y su familia se perpetuarán biológica y socialmente. De nuevo en palabras de Marx: "el consumo individual de la clase obrera es la operación por la cual los medios de subsistencia enajenados a cambio de fuerza de trabajo se reconvierten en fuerza de trabajo nuevamente explotable por el capital; es la producción y reproducción de su medio de producción más necesario: del obrero mismo" (2017: 662).

En un sentido parecido, en las últimas páginas de Salario, precio y ganancia (un panfleto breve de 1865 que Marx leyó en dos sesiones del Consejo General de la AIT como respuesta a las tesis de John Weston, un carpintero oweniano que defendía que las luchas por el aumento de los salarios eran, en última instancia, perjudiciales para el proletariado) Marx sugiere que el valor de la fuerza de trabajo oscila entre un límite mínimo, que se corresponde con el valor de los artículos con que el obrero se reproduce (él y, por descontado, su familia) y un límite máximo que "lo traza la fuerza física del obrero" (2007: 92). Límite mínimo, por tanto, que, según Marx (2007: 94), es el "límite mínimo del salario", expresado en un salario mínimo con el cual adquirir unos bienes mínimos para la subsistencia mínima del trabajador; y un límite máximo, que es el "máximo físico de la jornada laboral", expresado en un tiempo máximo por encima del cual no se podría (o debería) trabajar más. Se trata de dos límites que tienen que ver, exclusivamente, con la capacidad física del trabajador (hoy diríamos: con su cuerpo). Un límite mínimo, el del salario, que es un límite -positivo- a la reproducción del cuerpo; y un límite máximo, el de la jornada laboral, que es un límite -negativo- a la explotación del cuerpo. Se trata, en definitiva, de una horquilla por debajo de la cual el trabajador no puede tan poco y por encima de la cual el trabajador ya no puede más. Sobra decir, como repite Marx en varias ocasiones, que se trata de límites extremos, "aunque sean muy elásticos" (2007: 92) ${ }^{3}$. En ambos casos,

\footnotetext{
${ }^{2}$ Es más, el valor de la fuerza de trabajo es el valor de su (re)producción, que se identifica, a su vez, con el valor de los medios de subsistencia que emplea para reproducirse.

3 Es evidente que el cumplimiento de estos límites extremos no está, ni mucho menos, asegurado. Se trata de límites que no son naturales ni fijos sino, muy al contrario, históricos y
} 
por tanto, el interés lo fija el cuerpo, y no, como podría pensarse (sic.), la moral bondadosa del capitalista. Podemos expresarlo de la siguiente manera: los límites establecidos en la relación salarial son, por un lado, un límite mínimo para vivir y un límite máximo para no morir. Y ambos extremos de la horquilla, suelo y techo de la relación salarial, son un suelo y un techo físico: son el suelo y el techo del cuerpo.

Al margen de sus diferencias epistemológicas, se trata de dos esquemas explicativos que nos sitúan en un lado del juego de la reproducción -al menos tal y como ésta se dirimía a mediados del siglo XIX-, aquel que participa de la lógica de un contrato que, en términos generales, vincula cuerpo del trabajador, salario-consumo y jornada laboral. El otro lado de los ejercicios reproductivos de la fuerza de trabajo está ocupado, como se encargará de reivindicar la crítica feminista, por el trabajo de cuidados desempeñado por mujeres en el ámbito doméstico. La norma reproductiva (sus límites, sus variaciones, su ejercicio mismo) se decide, además de en las condiciones de trabajo remunerado, en las configuraciones privadas e invisibilizadas del trabajo no pagado de las mujeres. Se trata de unos estándares (re)productivos que la mujer modifica y padece. Como escribe Mariarosa Dalla Costa (2009: 31), "la mujer [...] se ha visto aislada en el hogar, obligada a desarrollar un trabajo considerado no cualificado, el trabajo de hacer nacer, criar, disciplinar y servir a la fuerza de trabajo de cara a la producción. En el ciclo de la producción social, su papel ha seguido siendo invisible, porque lo único visible es el producto de su trabajo, el obrero". Superponiendo, por tanto, a esta política de límites «masculinos», las variaciones históricas del trabajo reproductivo desempeñado por mujeres, intentaremos trazar una genealogía rápida de las oscilaciones en la norma reproductiva de los cuerpos a lo largo del siglo XX hasta llegar a las nuevas configuraciones de la reproducción bajo el neoliberalismo.

\section{LA POLÍTICA REPRODUCTIVA DEL FORDISMO}

En la historia social de estas reestructuraciones, la salarización de la reproducción que introduce el fordismo y la cobertura social welfarista marcarán el rumbo de la política corporal (reproductiva) hegemónica al menos hasta mediados de los años setenta. Hasta entonces -en tiempos de Marx, podríamos decir- los espacios reproductivos de la fuerza de trabajo discurrían entre un salario claramente insuficiente, un consumo poco generalizado (generalmente sustituido por prácticas de autoabastecimiento, sobre todo en zonas rurales) el trabajo de cuidados desempeñado por mujeres en el ámbito doméstico y una red asistencial promovida por el mutualismo sindical y la caridad religiosa. El Estado de pobres típico del siglo XIX, asistencial a niveles capilares y muy focalizados, centraba sus esfuerzos en cuestiones más relacionadas con el orden público que en garantizar la reproducción de la fuerza de trabajo. La íntima convivencia de pobreza y trabajo era considerada un fenómeno antes natural que transitorio, y aunque los ecos de esta confluencia desagradable resuenen hoy en

socialmente móviles. Es más, podría decirse que esta es la horquilla en la que se mueven históricamente las reivindicaciones obreras y los ajustes patronales. En este sentido, la lucha de clases dentro de la relación laboral capitalista podría leerse, siguiendo la metáfora marxiana, como la negociación histórica de los límites mínimos y máximos, de su dilatación, reestructuración o transgresión (Marx, 2017: 332). 
día, lo cierto es que, durante las décadas siguientes, el objetivo común de ingenieros industriales, empresarios y políticos fue (por motivos sin duda más tristes que alegres) consolidar su separación. Será a partir de los años 10, 20 y 30, primero con Ford y más tarde con Roosevelt, cuando el salario sea concebido (en la teoría y sobre todo en la práctica) como el medio principal y mayoritario para garantizar, mediante el consumo de bienes de subsistencia, la reproducción de los cuerpos. En este sentido, y recogiendo los términos que sugería Marx, la política de subidas salariales fordistas puede entenderse como una ampliación del límite mínimo salarial con la finalidad de reducir el límite máximo de la explotación ${ }^{4}$. La razón de este nuevo vínculo (otra vez más triste que alegremente) fue, en primer lugar, el potencial aumento de la productividad. En palabras del propio Ford, "pagando mal a los hombres preparamos una generación de niños subalimentados y subdesarrollados tanto física como moralmente; tendremos una generación de obreros débiles de cuerpo y de espíritu, que por esa razón se mostrarán ineficaces cuando entren en la industria. En definitiva,

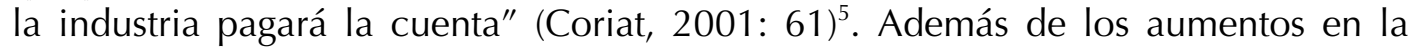
productividad y la intensidad del trabajo; además, también, de la consolidación de un mercado fluido de mano de obra disponible, Ford tenía en mente (como vanguardia de las aspiraciones patronales) la desarticulación progresiva de la labor asistencial de la que se encargaban los sindicatos y las cajas de socorro y de ayuda mutua. En la época, y a ojos de un empresario, la autonomía reproductiva de la clase trabajadora era directamente proporcional a su grado de desindicalización. En cualquier caso, lo relevante es la manera en que el pago de un salario deja de representar un estímulo al trabajo y pasa a consolidarse como el instrumento principal para sufragar la reproducción de los cuerpos trabajadores (Coriat, 2001: 53).

El 5 de enero de 1914 Ford anuncia una subida salarial de dos dólares y medio. Con respecto a la media, se trataba, ni más ni menos, del doble del salario diario habitual. La noticia del five dollar day llega a tantos hogares que la compañía tiene que filtrar las demandas por criterios geográficos y sólo aquellos que lleven un mínimo de seis meses residiendo en Detroit podrán beneficiarse del nuevo plan. Además de los habituales criterios productivistas (estabilizar los flujos de mano de obra, reducir el absentismo laboral y sobre todo reducir la altísima tasa de rotación) el five dollar day hace transparente como ninguna otra medida el afianzamiento definitivo -según lo que venimos sugiriendo- del nudo reproducción-salario. Es más, es su propia lógica interna -sus mecanismos de autovalidación, podríamos decir- la que expresa esta creciente intimidad (entre salario y reproducción de los cuerpos), pues los aumentos anunciados estaban sujetos y condicionados al cumplimiento de una serie de normas

\footnotetext{
${ }^{4}$ Lo cual no significa, como es evidente, que se redujera la intensidad del trabajo.

${ }^{5}$ Se trata de una cita recogida por Huw Beynon, trabajador de la Ford Motor Company que recogerá sus memorias fabriles en el célebre libro Working for Ford. Más allá de las apariencias, Ford es perfectamente consciente de la insuficiencia de los salarios de la época para costear la vida media de un hogar. Además, no es posible -comenta Ford- determinar matemáticamente los costes de "reposición de la fuerza del trabajador". Teniendo en cuenta que "abolir el sistema salarial y substituirlo por la propiedad comunal no ayudará a solucionar los problemas", las subidas salariales se presentan como la única solución (Ford, 2005: 232, 233) [Trads. nuestras.]
} 
reproductivas: no beber alcohol en casa, no abusar físicamente de los miembros de la familia, mantener la casa limpia, no subarrendar cuartos o destinar parte del salario a una cuenta de ahorros ${ }^{6}$. Como puede percibirse, se ponen en juego un conjunto de criterios domésticos cuya supervisión, encargada al recién creado Departamento de Sociología de la Ford Motor Company, presuponía, ni más ni menos, la intromisión directa en los ciclos reproductivos de una familia. No se trata tanto de garantizar un control ideológico de la mano de obra sino de ajustar la reproducción de los cuerpos a criterios morales y, sobre todo, económicos, tanto «externos»-relacionados con la economía de la empresa- como «internos»-relacionados con la economía doméstica. El ciclo salario-reproducción-consumo está ya perfectamente estandarizado y el «Model T» de Ford, retrato de un sueño americano esencialmente reproductivo, lo escenifica a la perfección.

Si bien parece un esquema reproductivo que promueve una mayor independencia de la población trabajadora, lo cierto es que, como recuerda Mariarosa Dalla Costa (2009: 31), "a través del salario se organizaba", también, "la explotación del trabajador no asalariado", pues la política fordista de salarización de la reproducción a través del consumo descansa sobre una consolidada e invisible división sexual del trabajo, donde el cabeza de familia o breadwinner, debe asegurar la reproducción de sus hijos y la de su mujer, homemaker encargada del trabajo doméstico. Se trata, por otra parte, de un ideal regulativo (el denominado "salario familiar») empírica y simbólicamente asumido al menos hasta los años ochenta del pasado siglo (Fraser, 2013: 111-135). Es fundamental comprender, en este sentido, que todo esquema de reproducción asalariada incluye formatos de trabajo reproductivo no asalariado, y que, por tanto, a toda «esclavitud del salario»-por usar la paráfrasis clásica para referirse a la ficción que subyace al libre intercambio de fuerza de trabajo en el mercado laboral- le corresponde una esclavitud del no-salario. En el marco reproductivo del fordismo, la creciente dependencia del salario y de los espacios de consumo mercantiles, convive, por tanto, con un conjunto de dependencias extralaborales, de "actividades residuales con respecto de las del mercado", por decirlo en los términos de Amaia Pérez Orozco (2017: 104), que podemos Ilamar cuidados y que, como veremos más adelante, subyacen como una constante invisible a los diferentes juegos reproductivos a lo largo del siglo XX y principios del XXI.

\section{EL NEW DEAL COMO INTERVENCIÓN EN LOS CRITERIOS REPRODUCTIVOS}

La consolidación de esta norma reproductiva por parte de la Ford Motor Company puede considerarse, en cierto modo, una anticipación managerial de las políticas

\footnotetext{
${ }^{6}$ La política administrativa del five dollar day es altamente eficiente: se parte de un salario base, diario y en función del puesto desempeñado (wage), al que se le añade un plus (profit) en función de la eficiencia y comportamiento reproductivo del trabajador. En términos reproductivos es importante destacar que se trata de una de las primeras homologaciones de salario por tarea desempeñada -lo cual sólo fue posible, en cierta manera, por la consolidación de la cadena de producción fordista- frente a las prácticas a destajo, las primas o el sistema de incentivos. Unido con la reducción de la jornada laboral de 9 a 8 horas, la política de subidas salariales fordistas terminó en un lapso relativamente breve de tiempo con las altas tasas de rotación y temporalidad laboral (Ford, 2005: 235 y ss.).
} 
keynesianas de demanda efectiva y de creación de un mercado interior que marcaron las décadas posteriores. El propio Ford aseguraba ya en 1922 que "si repartimos mucho dinero, ese dinero se gastará", lo cual, claro está, "se reflejará en un aumento de nuestras ventas" (2005: 233). Si bien en los años 20 el control de los ciclos reproductivos de la clase trabajadora dependía casi exclusivamente de un salario directo y una política consumista crecientes (junto con la habitual participación en las Ilamadas cajas de seguro, dirigidas por los sindicatos y algunos sectores patronales y, de nuevo, el invisibilizado trabajo doméstico desempeñado por mujeres) no será hasta el New Deal (1933-1938) cuando el Estado comience -al menos en EE.UU.- a asumir entre sus principales obligaciones el cuidado reproductivo de los trabajadores. Vale la pena repasar, aunque sea rápidamente, la famosa sección séptima de la National Industry Recovery Act (NIRA) ${ }^{7}$. Se trata de una sección dedicada en su mayor parte a la protección de la libertad sindical (con clausulas restrictivas o prohibitivas para los empleadores ${ }^{8}$ ), a la promoción de la participación sindical de los trabajadores ${ }^{9}$ y a blindar jurídicamente la regulación de las condiciones laborales. Este último punto es fundamental. Según reza el tercer artículo, "los empleadores deberán cumplir con el máximo de horas de trabajo, los mínimos porcentajes salariales y otras condiciones laborales aprobadas o prescritas por el presidente" (NIRA, 1933: Section 7.a). Y en una cláusula posterior: "cuando no se llegue a un acuerdo mutuo [entre empleados y empleador] [...] el Presidente tendrá la autoridad para fijar un código de competencia leal que fije el máximo de horas de trabajo, el mínimo de cuantías salariales y otras condiciones laborales" (NIRA, 1933: Section 7.c). Observamos, por tanto, una formalización jurídica -sin duda primitiva- de lo que, ya con Marx, hemos identificado como el techo y el suelo de la política corporal o reproductiva en el ámbito laboral remunerado: un límite mínimo del salario y un límite máximo de la explotación, ambos ahora institucionalizados -al menos de derecho- y previsiblemente regulados colectiva o sindicalmente; además, esta codificación de los límites en el ámbito laboral se acompañará de la creación de un sistema contributivo de pensiones que garantiza la jubilación a partir de los 65 años y promueve seguros por desempleo y minusvalía (Social Security Act de 1935). En cierto modo, los años 30 del pasado siglo marcan la «década cero» del

\footnotetext{
${ }^{7}$ Se trata de la primera gran ley del New Deal, aprobada por el gobierno Roosevelt en 1933, más tarde declarada inconstitucional por la Corte Suprema en 1935 y en cierto modo reemplazada por la National Labor Relations Act, la cual incluía los artículos relacionados con las libertades sindicales presentes en la sección a la que nos referimos.

${ }^{8}$ Los cuales no podrán "interferir restrictivamente" ni "coaccionar" a los empleados en la elección de los representantes sindicales, en su "autoorganización" o en otras actividades relacionadas con la "negociación colectiva" (National Industry Recovery Act, 16 junio 1933, Section 7.a). Del mismo modo, tal como reza el segundo artículo, no se le podrá exigir a ningún empleado unirse a un sindicato amarillo (company union) ni impedirle su participación en un sindicato de su propia elección. [Trads. nuestras]

${ }^{9}$ Los empleados tienen "derecho a organizarse y negociar colectivamente a través de representantes de su propia elección" (NIRA, Section 7.a).
} 
intervencionismo estatal en cuestiones que incumben a la reproducción de la fuerza de trabajo ${ }^{10}$.

Como indica Mariarosa Dalla Costa, la racionalización reproductiva que representa el New Deal fue, al mismo tiempo, una planificación meditada de las tareas y responsabilidades de la mujer en el ámbito doméstico. Se revaloriza la figura del ama de casa, que deberá encargarse exclusivamente de la reproducción de la fuerza de trabajo y no, como podía ocurrir en las décadas anteriores, también a la producción de bienes autoconsumibles o para el comercio a pequeña escala; se intensifican y amplían las tareas domésticas (entre otras cosas por la aparición de un sinfín de aparatos e inventos social y simbólicamente necesarios); se acentúa la moralización del trabajo doméstico, identificando la gratuidad de las tareas con el amor incondicional, etc. (Dalla Costa, 2009: 157-159). La alarma social creada al calor de la Gran Depresión alrededor de la coreadísima «disgregación de la familia» (por los numerosos divorcios, los bajos índices de natalidad o las crecientes tentativas de independencia laboral), unido con la necesidad de estabilizar los flujos de mano de obra disponibles y aumentar el bienestar de la fuerza de trabajo productiva, harán de la familia la unidad económica básica. En palabras de Dalla Costa: "lo que se le demanda a la mujer es, en primer lugar, la responsabilidad de garantizar el sostenimiento real del crecimiento salarial" -es decir, el poder adquisitivo de la familia- pero también "la reabsorción y reproducción de los individuos que no son inmediatamente fuerza de trabajo" -los desempleados- y, por descontado, la "producción adecuada de nueva fuerza de trabajo y [...] [la] reproducción de la fuerza de trabajo ya existente" (2009: 249-250).

Como resultado de la desfavorable relación de fuerzas con la que los Estados capitalistas terminan la II Guerra Mundial (en relación con la pujanza del movimiento

\footnotetext{
${ }^{10} \mathrm{Si}$ bien Estados Unidos representa un ejemplo privilegiado para captar las variaciones a las que nos referimos, se trata de un proceso rastreable en otros países. En Francia será bajo el gobierno del Frente Popular cuando se comience a proteger y asegurar la reproducción de la fuerza de trabajo con la juridificación de la relación laboral mediante la homologación de los convenios colectivos, la promoción de las libertades sindicales y los aumentos salariales, tal y como se recoge en los Acuerdos de Matignon (producto de las huelgas que tuvieron lugar en mayo y junio -no del 68 sino del 36- y que recogen, por primera vez en el derecho laboral francés, la legalización de los convenios colectivos, la libertad de participación y delegación sindical y aumentos salariales porcentuales. Al margen de estos acuerdos, y en aplicación del programa de gobierno del Frente Popular, se reducirá la jornada laboral semanal a 40 horas y se oficializarán las vacaciones pagadas (Les Accords Matignon, 7 junio 1936). En España, la "República democrática de trabajadores" -como reza el primer artículo de la Constitución del 9 de diciembre de 1931- constitucionalizará la libertad sindical y los derechos del trabajador (Constitución de la República Española, 9 diciembre 1931). México y Alemania, por mencionar dos de los casos más notables, harán lo propio una década antes.
} 
obrero), la denominada "ciudadanía laboral ${ }^{11}$ de los Estados de bienestar marcará la norma reproductiva al menos durante los Ilamados treinta gloriosos ${ }^{12}$.

\section{LA POLÍTICA REPRODUCTIVA DE LA CIUDADANÍA LABORAL WELFARISTA}

La idea de ciudadanía laboral (un concepto cuyo «sentido» es, por lo general, muy posterior a su «referencia», en la medida en que acostumbra a utilizarse para hablar de su pérdida o entrada en crisis $^{13}$ ) expresa la unión -más bien el resultado de la unión- del pacto de clases en materia laboral (una regulación institucionalizada de derechos y deberes para empleados y empleadores) y la intervención activa del Estado en la economía (que pasa a ser un agente distribuidor de renta). Los efectos de esta suerte de contrato social serán, de un lado, la relación laboral estable (la seguridad en el puesto de trabajo y, por lo general, la constitucionalización de la negociación colectiva) y el disfrute universal de los derechos de segunda generación (organizados como un sistema de seguros sociales contributivos). Ambas condiciones o espacios de esta noción de ciudadanía -la política laboral y la política social- son recíprocamente dependientes y, como sugiere Luis Enrique Alonso (1999: 9), consisten en un "modo de integración centrípeto y ordenado de la reproducción de la fuerza de trabajo". De hecho, el nuevo cuerpo jurídico laboral que promueve este tipo de ciudadanía es, dicho rápidamente, un cuerpo de garantías principalmente reproductivas o materiales ${ }^{14}$, de igual manera que los derechos sociales o la idea misma de Estado de bienestar. Visto desde otro punto de vista, se trata de un pacto entre clases por el cual se negocian y -como en todo pacto- se ceden, una serie de derechos a cambio de una serie de contribuciones. Es importante distinguir, en esta línea, entre estas políticas y Estados de bienestar y las viejas políticas y Estados asistenciales: si bien estos últimos asistían a aquellos que, temporal o indefinidamente, se situaban fuera de la relación y el circuito salarial (recordemos: la reproducción de los cuerpos se costeaba casi en exclusiva mediante el salario directo), el pacto de bienestar o ciudadanía laboral se dirige a los que -dicho rápidamenteestán dentro, es decir, a los que contribuyen ${ }^{15}$. Se trata de una diferencia fundamental

\footnotetext{
${ }^{11}$ También denominada «ciudadanía social», aunque, en rigor, se trata de un concepto más amplio, pues junto con el empleo formal (ciudadanía laboral), la nacionalidad y la pertenencia a una familia patriarcal son igualmente vías de acceso a la ciudadanía social (Alonso, 2007: 15 y ss).

${ }^{12}$ La expresión es del economista Jean Fourastié y abarca el período comprendido entre el fin de la segunda guerra mundial y, aproximadamente, la crisis del petróleo de 1973.

${ }^{13}$ Aunque es difícil rastrear los primeros usos de esta locución, lo cierto es que aparece en el vocabulario político y jurídico a partir de los años ochenta en Europa y de los noventa en Latinoamérica (Muñoz León, 2013).

14 Puede hablarse, en este sentido, de "ciudadanía material" e incluso de "socialdemocratización material de los modos de vida" (Alonso, 1999: 9-10).

${ }^{15}$ Como escribe Alonso (2006: 113), "en el ciclo que se inicia en la salida de la Segunda Guerra Mundial, el trabajo es inseparable de su juridificación pública (superando la relación contractual privada) y, por tanto, de su conversión en un derecho de ciudadanía que asociaba titularidades y bienes públicos".
} 
para comprender la sucesión y creación de responsabilidades en la reproducción de los cuerpos bajo el capitalismo. En este sentido, podría decirse que, así como la asistencia regula o controla la exclusión, el bienestar regula o controla la desigualdad ${ }^{16}$.

Por muy breve que sea, ninguna reconstrucción sobre el sentido de ciudadanía social puede pasar por alto las conferencias que T. H. Marshall pronunció en 1949 (publicadas un año más tarde con el título Ciudadanía y clase social) ${ }^{17}$ acerca de la evolución histórica de la ciudadanía y los derechos otorgados civiles (siglo XVIII), políticos (siglo XIX) y sociales (siglo XX). Si bien se trata de un esquema que no está de más recordar, lo cierto es que la escala evolutiva -casi lineal- que presenta es también una fotografía incómodamente fiel y transparente de un marco de sentido cuanto menos androcéntrico. Nancy Fraser se refiere explícitamente a ello cuando, al investigar la articulación social y mitológica de la ciudadanía civil (con la retórica del contrato y la independencia) y la social (identificada, por el contrario, con la dependencia caritativa) observa en la periodización de Marshall un ocultamiento de las desigualdades raciales y de género. En sus propias palabras, "al asumir, como harán más tarde las socialdemocracias, que los objetivos principales de la ciudadanía social son erosionar la desigualdad de clase y protegerse frente a las fuerzas del mercado, se desplazan otros focos de desigualdad fundamentales, así como otros mecanismos y espacios de dominación" (Fraser, 1992: 50). Por lo demás, se trata de un lugar común en las prácticas y discursos de la ciudadanía social y laboral: es la historia social -única y directa- del varón blanco contribuyente ${ }^{18}$. Por el contrario, la titularidad de los derechos (la familia, donde el hombre asalariado es titular de derechos directos, mientras que la mujer ama de casa es titular derivada), la base de acceso a los mismos (las prestaciones contributivas de la población asalariada y no de la ciudadanía en su conjunto) o su contenido (derecho a la salud, a la educación y a la cobertura por desempleo pero no el derecho de cuidados a las personas dependientes, dando por hecho que son las mujeres quienes, de iure, los cubren) nos muestran, como recoge Amaia Pérez Orozco (2017: 140-151), la consustancialidad entre Estado de bienestar y división sexual del trabajo. En definitiva, si bien en los

\footnotetext{
${ }^{16}$ Para las diferencias entre exclusión y desigualdad, véase Boaventura de Sousa Santos (2011: 133-171).

17 El cual, nunca está de más recordarlo, no debe confundirse con el economista Alfred Marshall, cuyos Principios de economía (1890) marcarán notablemente las teorías de autores como Pigou o Keynes y que, (de ahí la posible confusión), por un lado, da nombre a las conferencias a las que nos referimos y, además, es el autor más citado en el ensayo de T. H. (Marshall; Bottomore, 2007).

${ }^{18}$ Historia que participa de lo que Antonella Picchio llama "estrabismo productivista", presente en aquellos análisis que reducen los conflictos sociales y laborales al conflicto capital-trabajo asalariado, excluyendo consideraciones sobre la importancia del trabajo no remunerado desempeñado por mujeres (Orozco, 2017). En cierto modo, centrar el análisis de la reproducción de la fuerza de trabajo en el consumo es, también, una forma de invisibilizar el trabajo reproductivo no pagado. Refiriéndose al reduccionismo de este mismo marco de sentido, Tithi Bhattacharya se refiere a la "visión del secretario general del sindicato" (2017: 89).
} 
Estados de bienestar la familia ya no representa en exclusividad el "universo reproductivo", no por ello deja de ser, como escribe Dalla Costa (2009: 233), un "polo necesario e invariante con respecto a las posibilidades de ejercicio de funciones reproductivas por parte del propio Estado". Sin duda se trata de una complementariedad viciosa que los nostálgicos del welfarismo incondicional deberían, cuanto menos, tener en cuenta.

Recogiendo brevemente lo expuesto hasta este punto, podemos concluir, por un lado, que cuando la reproducción de la fuerza de trabajo depende sobre todo de un salario, la mujer depende del trabajador que depende del salario; por otro lado, que cuando la reproducción se sufraga entre un salario y el disfrute de unos servicios sociales, la mujer también depende del trabajador que contribuye. Si bien la entrada en crisis del proyecto de la ciudadanía laboral y el advenimiento de las sociedades postindustriales conservará algunas de estas dependencias estructurales, es posible rastrear ciertas transformaciones fundamentales en las políticas de reproducción de los cuerpos.

\section{LA ECONOMÍA REPRODUCTIVA NEOLIBERAL}

No hace falta dar muchos rodeos -al menos no tantos como hasta ahora- para adentrarse en un asunto más o menos bien acotado, más o menos bien conocido, como es el neoliberalismo. Aunque seguramente para unos signifique más unas cosas (el ambicioso proyecto económico de ciertos personajes políticos, con rostros, programas y aspiraciones bien reconocibles) y para otros más bien otras (dicho pronto y mal: una racionalidad de gobierno dirigida a "conducir conductas" -Foucault, 1988: 15-, que implica una determinada producción de subjetividad y cuyas premisas y efectos no son ni por asomo reducibles a políticas o economías coyunturales), lo cierto es que «neoliberalismo» suele ser más un lugar común accesible que un no-lugar desconocido, con todas las «mediaciones» o «interrupciones» que la crítica presupone. En cualquier caso, también es cierto que entre estas mediaciones pocas veces (o algunas, pero tangencialmente) se atiende a la particularidad del neoliberalismo con respecto a la razón de gobierno previa, que, según lo que hemos comentado hasta ahora, sería propia de los Estados de bienestar y cuya forma social sería la ciudadanía laboral. Menos aún -hay que recordarlo- cuando se sitúa el peso analítico en ese espacio límite que nosotros hemos llamado cuerpo pero que, con Marx, podemos seguir Ilamando (siempre que tengamos en cuenta la connotación reduccionista de este término, al menos en su uso histórico o marxista) fuerza de trabajo. Es más, una de las particularidades de la gubernamentalidad neoliberal (de su proceso de producción de subjetividad articulado en torno a una serie de políticas concretas) aflora precisamente cuando se aborda su estudio desde el prisma del cuerpo en general o, como en el caso concreto que aquí abordamos, desde la reproducción de la fuerza de trabajo. De nuevo sin rodeos, es fácil observar en las políticas y discursos neoliberales una reestructuración radical de los cauces reproductivos, dentro y fuera del ámbito laboral, y en un doble sentido: por un lado, en el nivel de las políticas concretas, como un proceso de privatización de la reproducción de los cuerpos (que analizaremos en el epígrafe 5.1); por otro lado, en el nivel de la producción de discurso, - de la mitología teórica del neoliberalismo- 
como un borrado del «territorio» reproductivo y el «suelo» corporal de la fuerza de trabajo (5.2).

\section{La política reproductiva del neoliberalismo}

Lejos de lo que podría pensarse a simple vista, la progresiva desaparición de los welfare states desde mediados de los años setenta es, antes que una desaparición, una sustitución o -si se quiere- una reestructuración meditada de sus funciones clásicas. Si bien la particularidad de la ciudadanía laboral en los Estados capitalistas de bienestar era la derivación directa de los «derechos sociales de bienestar» con respecto a los «derechos colectivos del trabajo», los denominados workfare states neoliberales deben entenderse, ante todo, como un proyecto de escisión entre «políticas sociales» y «políticas laborales» (Alonso, 1999: 20). Se trata de una política económica y una economía política de ruptura entre ambas esferas: de un lado, las mínimas políticas sociales, cuyo público ya no es la ciudadanía laboralmente integrada sino, como en los antiguos Estados asistenciales, los grupos excluidos de la relación salarial; de otro lado, un conjunto de políticas laborales destinadas mayoritariamente a la población desempleada, con el fin de fomentar la empleabilidad y engrasar el mercado laboral con perfiles adaptados a exigencias y condiciones ya existentes. Es fundamental entender, en relación a este último punto, la perfecta (y servil) simbiosis entre política laboral y mercado laboral o, en otras palabras, entre programas institucionales de integración laboral y exigencias del mercado de trabajo. Sobra recordar que, históricamente, los estados de las sociedades capitalistas han representado un punto de apoyo imprescindible para el correcto funcionamiento de una serie de procesos productivos no precisamente consensuados en el parlamento. Pero si bien esto es innegable, también lo es que las Constituciones y códigos jurídicos de gran parte de los Estados occidentales al menos desde los años cuarenta, han supuesto, en materia laboral, un freno legal a las demandas y exigencias de tales procesos productivos. En este sentido, la política de los estados neoliberales debe pensarse como un ejercicio de adaptación absoluta a las exigencias -paradójicamente adaptativas- de los mercados de trabajo. Quien mejor ha entendido esta complementariedad viciosa es, sin duda, Jamie Peck, para el cual los workfare states representan no sólo el resultado del desmantelamiento progresivo de los Estados de bienestar, sino un programa (re)activo (constructivo, podría decirse ${ }^{19}$ ) con fines particulares y, sobre todo, originales. No implican, en este sentido, "una simple 'desregulación' de los mercados de trabajo [...] sino que promueven nuevas formas de intervención estatal" (Peck, 2003: 80) ${ }^{20}$ con el objetivo de fomentar la empleabilidad y la inclusión en el mercado laboral de los grupos sociales más desfavorecidos. Así -escribe Jamie Peck (2003: 85)- "en contraste con la lógica

\footnotetext{
${ }^{19}$ Es fundamental, en este sentido, entender el neoliberalismo como un proyecto que produce continuamente "las condiciones de la competencia" frente al "antiguo modelo liberal [...] del «dejar hacer, dejar pasar» que atiende "a las normas naturales del comercio". Se trata de dos modelos -uno que podríamos Ilamar constructivista y otro más bien naturalista-, que determinan, según lo que venimos sugiriendo, funciones específicas para sus órganos de gobierno (López Álvarez, 2010: 49).

${ }^{20}$ Trads. nuestras.
} 
welfarista de proporcionar 'refugios' temporales fuera del mercado de trabajo para determinados grupos sociales, [...] [la] lógica workfarista moviliza ciertos grupos sociales hacia dentro del mercado laboral, donde se espera que permanezcan indefinidamente, sin tener en consideración problemas sistémicos como el subempleo, las reducciones salariales o las relaciones de explotación laboral".

Recuperando el hilo de lo que venimos analizando, cabe preguntarse cómo afecta la proliferación de los workfare states al sistema de la reproducción de la fuerza de trabajo. En términos generales, la desaparición de las políticas sociales de bienestar implica una creciente privatización feminizada de la reproducción, tal y como sugieren autoras como Amaia Pérez Orozco (2017: 70, 146, 205), Sandra Ezquerra (2011) o Nancy Fraser (2013: 67-68) o, en términos generales, una crisis reproductiva sin precedentes ${ }^{21}$. En un escenario con escasos cuidados sociales, la reproducción de los cuerpos retorna con fuerza a la esfera privada, aumentando las demandas reproductivas en el ámbito doméstico y penalizando doblemente a aquellas mujeres que se incorporan al mundo laboral. De la misma manera, en un contexto postcontractual donde la socialización de los riesgos pierde vigencia (discursiva y material) y es sustituida por políticas y deseos infligidos de individualización de los peligros, el espacio clásico de la reproducción se hace, de facto y de iure, hiperresponsable de lo que es socialmente insuficiente. Este desplazamiento en la responsabilización (del Estado al ámbito privado del que nunca había salido del todo) "penaliza -en palabras de Wendy Brown (2016: 142)- de modo excepcional a las mujeres en la medida en que siguen siendo desproporcionadamente responsables de aquellos que no pueden ser responsables de sí mismos". A ojos de la política económica del neoliberalismo, la privatización de la reproducción a la que nos referimos supone, antes que nada, un ahorro. En este sentido, como han investigado autoras como Cristina Morini (2014) o Verónica Gago (2015: 99 y ss.), la feminización del trabajo de los últimos años no debe leerse exclusivamente desde parámetros cuantitativos sino, sobre todo, como sinónimo de la creciente asimilación por parte del capital del tipo de tareas tradicionalmente desempeñados por mujeres ${ }^{22}$. Se produce, por tanto, una doble privatización feminizada de la reproducción: una reprivatización espacial (del Estado a los hogares) para los que pueden menos y una privatización económica (de los hogares al mercado) para los que pueden más. En términos generales, se trata de un proceso por el cual se socializan "los riesgos del proceso de acumulación de capital mediante la re-privatización de los riesgos del

\footnotetext{
${ }^{21}$ Es el caso de Silvia Federici, que se refiere al neoliberalismo como un "ataque a la reproducción" (2013: 145) y, en términos económicos, lo identifica con un proceso de desinversión generalizado en fuerza de trabajo (2013: 168 y ss.) o -de nuevo- de Nancy Fraser, que habla de "crisis de los cuidados" (2017: 21) y de Amaia Pérez Orozco, que habla de intensificación del "conflicto capital-vida" (2017: 65)

22 "El trabajo vivo reproductivo, en la medida en que ha permitido reducir el coste de la fuerza de trabajo, es decir, el salario que se necesita para vivir, [...] ha permitido aumentar el beneficio del capital [...] El trabajo gratuito, en la era de las prácticas masivas sin remuneración, ha pasado de ser un bagaje atávico de las mujeres a transformarse en una experiencia común que atraviesa géneros y profesiones" (Morini, 2014: 141).
} 
proceso de sostenibilidad de la vida", por expresarlo en los términos de Amaia Pérez Orozco (2017: 116).

En cualquier caso, el trabajo de cuidados no pagado y desempeñado por mujeres no es el único espacio de privatización neoliberal de la reproducción. En cierto modo, las nuevas configuraciones internas a la relación laboral remunerada pueden leerse también como parte del proceso de (re)privatización reproductiva al que nos referimos. Sin duda el mejor ejemplo de ello lo encontramos en la desprotección (jurídica, social, reproductiva) generalizada de un colectivo laboral relativamente reciente pero ampliamente extendido como son los trabajadores autónomos de segunda generación. A este respecto, los intentos de Sergio Bologna por definir un estatuto de esta forma de trabajo no asalariado representan una guía imprescindible. Como sugiere el investigador italiano, la expansión de población trabajadora no asalariada revela ya un desplazamiento en la responsabilidad reproductiva, "sanciona[n]do por primera vez de facto el principio de que la subsistencia de la fuerza de trabajo ya no es un problema del que el empleador o el Estado deban hacerse cargo" (Bologna, 2006: 72). Al sustituir la forma "salario» por una retribución en forma de factura cuyos plazos de pago no están jurídicamente establecidos ${ }^{23}$, el espacio de la responsabilidad o garantía reproductiva se hace exclusivamente individual. Simplemente, se privatiza. En palabras de Bologna:

[se] suprime de golpe el problema de la reproducción de la fuerza de trabajo como problema constitutivo de las relaciones sociales y, por lo tanto, de las relaciones contractuales entre patrón y trabajador y de las relaciones de ciudadanía entre trabajador y Estado. Se trata de un cambio radical, mediante el cual el principio fundamental de garantía de la subsistencia se sustituye por la condición de facto de riesgo existencial (2006: 72).

Al desaparecer la retribución contractual clásica ( $x$ número de horas a cambio de y salario para reproducir la fuerza de trabajo; asunto, por otro lado, ya perfectamente esquematizado en El Capital) desaparece, antes que nada, la garantía de supervivencia de la fuerza de trabajo misma. La socialización institucionalizada del riesgo que representaba el proyecto de una ciudadanía laboral está ya completamente resquebrajada y las condiciones de existencia de los cuerpos que trabajan se deciden, cada vez más, dentro de las fronteras del mercado. Con todo, la falta de codificación jurídica de estas nuevas formas de empleo es plenamente solidaria de los procesos de desregulación de la relación laboral asalariada, donde la inestabilidad salarial y la extensión de la jornada laboral social (los límites mínimos y máximos a los que se

\footnotetext{
${ }^{23}$ Se trata de una actualización recrudecida de la deuda que, como Marx observaba, todo empleador contrae al alquilar fuerza de trabajo a cambio de un salario: es el obrero quien "adelanta al capitalista el valor de uso de la fuerza de trabajo [y] le permite al comprador que la consuma antes de haber recibido el pago del precio correspondiente. En todas partes es el obrero el que le da crédito al capitalista" (Marx, 2017: 233). Sobra recordar que, en el caso del trabajador autónomo, no hay siquiera garantía de que ese «crédito» o "adelanto» sea recompensado dentro de unos márgenes temporales.
} 
refería Marx) son, antes que una situación transitoria, la nueva norma de un mercado laboral precarizado ${ }^{24}$.

Ciertamente, las nuevas políticas reproductivas del neoliberalismo se mueven, además, en un marco simbólico que prioriza la autonomía y «autoapreciación» (Feher, 2009) de los sujetos sobre las condiciones materiales de su realización. En este sentido, es importante atender también, aunque sea brevemente, a los discursos de (auto)legitimación de tales prácticas reproductivas.

\section{El discurso reproductivo del neoliberalismo}

La progresiva desaparición de las políticas sociales de bienestar, que nosotros identificamos con una re-privatización de la reproducción a varios niveles, implican también, y en cierto sentido, una regresión a la salarización de la reproducción (que ya observamos en las primeras décadas del siglo XX), o, lo que es lo mismo, una vuelta a la (hiper)dependencia del salario en la reproducción de los cuerpos. Se trata de una dependencia salarial que paradójicamente, y por ser el reverso de la «dependencia welfarista» o asistencial contra la que se construye discursivamente el neoliberalismo ${ }^{25}$, es presentada más bien (al menos desde las postrimerías del 68) en términos de independencia o, al menos, de autonomía y libertad individual. Como ya comentamos anteriormente, y más allá de su propia mitología, las políticas workfaristas funcionan antes como herramientas adaptativas a un mercado laboral rotatorio y precario que como ayuda externa frente a la inestabilidad... ¡de estos mismos mercados! La (hiper)salarización de la reproducción no debe entenderse, en este sentido, como un potencial espacio de decisión irrestricta sino, muy al contrario, como el efecto peligroso de una renuncia, la del Estado, a garantizar unos niveles mínimos de reproducción y cuidado de los cuerpos.

Paradójicamente, esta clase de renuncia (que acostumbra a presentarse como un indulto a las excesivas responsabilidades históricas de los Estados) afecta también, al menos discursivamente, a la idea misma de salario. Foucault lo comenta en una de sus clases del Collège de France (14 marzo 1979) cuando, asumiendo la posición que adoptarían los economistas neoliberales Theodore Schultz o Gary Becker, escribe:

"¿qué es un salario? [...] Desde el punto de vista del trabajador, el salario no es el precio de venta de su fuerza de trabajo, es un ingreso [...] ¿qué es

\footnotetext{
${ }^{24}$ La proliferación de los minijobs, los working poors o los falsos autónomos dan buena cuenta de ello. Asimismo, el fenómeno del desempleo y de producción de «poblaciones superfluas» (Briales, 2017) ofrecen una perspectiva fundamental para entender la política laboral y reproductiva del neoliberalismo (la cual, por cuestiones de espacio, no desarrollaremos en este texto).

${ }^{25}$ Resulta interesante, en este sentido, atender a las variaciones que registra Nancy Fraser en torno a los discursos sobre la dependencia en i) la era preindustrial, donde la dependencia está relacionada con trabajar para otra persona, ii) el capitalismo industrial, donde la dependencia se identifica, paradójicamente, con los excluidos de la relación laboral, iii) el capitalismo de bienestar, donde la dependencia se identifica con ser receptor de una ayuda y iv) la sociedad postindustrial, donde la dependencia se individualiza e incluso patologiza (Fraser, 2013: 83-110).
} 
un ingreso? [...] Un ingreso es sencillamente el producto o rendimiento de un capital. Y a la inversa, se denominará capital todo lo que pueda ser de una manera u otra fuente de ingresos futuros. Por consiguiente, sobre esa base, si se admite que el salario es un ingreso, el salario es por lo tanto la renta de un capital. Ahora bien, ¿qué es el capital cuya renta es el salario? Bueno, es el conjunto de los factores físicos, psicológicos, que dan a alguien la capacidad de ganar tal o cual salario, de modo que, visto desde el lado del trabajador, el trabajo no es una mercancía reducida por abstracción a la fuerza de trabajo y el tiempo [durante] el cual se lo utiliza. Descompuesto desde la perspectiva del trabajador en términos económicos, el trabajo comporta un capital, es decir, una aptitud, una idoneidad" (2012: 226).

Por lo pronto, puede observarse una mutación fundamental -al menos "desde el punto de vista del trabajador", como dice Foucault- con respecto a las definiciones de salario y fuerza de trabajo que vimos con Marx y que se consolidan de una u otra manera en las diferentes fases del sistema de trabajo asalariado. El salario, que es la expresión monetaria del valor de la fuerza de trabajo (mensurable por el valor de los bienes de subsistencia necesarios para su reproducción) ya no es, para el trabajador, el precio de venta de su fuerza de trabajo, como tampoco es, en términos vulgares, el dinero empleable en los gastos de su subsistencia, sino, muy al contrario, el rendimiento de un capital. Ya no es, en definitiva, un precio que se paga a cambio de algo (el alquiler de la fuerza de trabajo) para reponer otro algo (esa misma fuerza de trabajo; un cuerpo) sino, por lo pronto, el ingreso que produce una determinada "aptitud". Al salario, por tanto, ya no le incumbe el cuerpo sino más bien el desarrollo de una "idoneidad" que, en definitiva, valga la pena. Como vemos, en el marco de sentido neoliberal, igual que ocurrió con el Estado, el salario pierde su vínculo con la reproducción y lo gana, de nuevo, con el capital. Parecería, por tanto, que la única fuente de renta que contempla la racionalidad neoliberal es la de un capital y no, como hasta ahora hemos sugerido, un cuerpo. Estado y salario aparecen, desde este punto de vista, transformados en sus funciones clásicas y se convierten, a su manera, en capital. El trabajo, continúa Foucault (2012: 227), se descompone para Schultz y Becker en capital y renta. La renta es el salario; el capital es el trabajador. Aunque para nosotros siga siendo lo mismo, los cuidados de una fuerza de trabajo que es "músculo, nervio y cerebro" y los de un "capital humano» no son, institucional ni simbólicamente, los mismos ${ }^{26}$.

\footnotetext{
${ }^{26}$ Resulta relativamente sencillo encontrar variaciones de esta nueva norma en los panfletos vanguardistas de las actuales técnicas de management empresarial, donde se promueve el denominado «salario emocional» como complemento ( $y$ a veces sustituto) de la, por otra parte, ya aburrida y anticuada (sic.) retribución salarial clásica. Se trata de una cristalización más del desplazamiento del welfare al Ilamado wellness, un bienestar que "no consiste en la cobertura de carencias y necesidades [sino que] se identifica con la maximización de las capacidades y competencias individuales, [...] especialmente con las emocionales y afectivas" (Vázquez García, 2017: 7).
} 


\section{CONCLUSIONES}

Recogiendo las variaciones registradas en la reproducción de los cuerpos hasta el presente, podemos concluir que i) en tanto que economía política cristalizada en medidas y acciones concretas, el neoliberalismo puede entenderse como una privatización espacial, jurídica y económica de la reproducción. Espacial, pues el sujeto responsable de la reproducción ya no es semipúblico (el Estado y la mujer) sino privado (el individuo y, por consiguiente, el hogar); jurídica, pues ya no hay una codificación institucionalizada que regule la reproducción (por la desregulación de la relación asalariada y la obsolescencia jurídica con respecto a nuevas formas de empleo) sino que ésta se juega más bien entre pactos y criterios interpersonales o privados; económica, pues en algunos casos las prestaciones sociales son sustituidas por servicios en el mercado. Si hay algo que estas realidades tienen en común, es sin duda el desplazamiento de la socialización a la privatización o individualización de los riesgos sociales. Asimismo, ii) que en tanto que producción de un discurso y un marco de sentido propio, el neoliberalismo opera como un borrado de las dependencias y sujeciones corporales que acompañan todo proceso de reproducción social. Ambos aspectos son, en cierto sentido, expresiones de una misma norma que sitúa al cuerpo en un horizonte reproductivo cuanto menos inseguro.

\section{BIBLIOGRAFÍA}

ALONSO, Luis Enrique. (1999). "Crisis de la sociedad del trabajo y ciudadanía: una reflexión entre lo global y lo local". Política y Sociedad, 31, pp. 7-35.

- (2006). "Centralidad del trabajo y cohesión social: ¿una relación necesaria?". Gaceta sindical: reflexión y debate, 7, pp. 101-126.

- (2007). La crisis de la ciudadanía laboral. Barcelona: Anthropos.

BHATTACHARYA, Tithi. (2017). "How Not to Skip Class: Social Reproduction of Labor and the Global Working Class", en BHATTACHARYA, Tithi (ed.) (2017). Social Reproduction Theory. Remapping Class, Recentering Oppression. Londres: Pluto Press.

BOLOGNA, Sergio (2006). Crisis de la clase media y posfordismo. Madrid: Akal.

BRIALES, Álvaro (2017). "Entre el ejército de reserva y el tiempo superfluo: actualizaciones para una interpretación del desempleo en la teoría crítica de Marx". Constelaciones: Revista de Teoría Crítica, 8/9, pp. 367-388.

BROWN, Wendy (2016). El pueblo sin atributos. La secreta revolución del neoliberalismo. Barcelona: Malpaso.

CORIAT, Benjamin (2001). El taller y el cronómetro. Ensayo sobre el taylorismo, el fordismo y la producción en masa. Madrid: Siglo XXI.

DALLA COSTA, Mariarosa (2009). Dinero, perlas y flores en la reproducción feminista. Madrid: Akal. 
EZQUERRA, Sandra (2011). “Crisis de los cuidados y crisis sistémica: la reproducción como pilar de la economía Ilamada real". Investigaciones Feministas, 2, pp. 175194.

FEDERICl, Silvia (2013). Revolución en punto cero. Trabajo doméstico, reproducción y luchas feministas. Madrid: Traficantes de Sueños.

FEHER, Michel (2009). "Self-Appreciation; or, The Aspirations of Human Capital". Public Culture, 21 (1), pp. 21-41.

FORD, Henry (2005). My life and work. Consultado el 19 de octubre de 2018, The Project Gutenberg, en http://www.gutenberg.org/ebooks/7213.

FOUCAULT, Michel (1988). "El sujeto y el poder". Revista Mexicana de Sociología, 50 (3) pp. 3-20.

- (2012). Nacimiento de la biopolítica. Madrid: Akal.

FRASER, Nancy (1992). "Contract versus Charity. Why is there no Social Citenzship in the United States?". Socialist Review, 22 (3), pp. 45-67.

- (2013). Fortunes of feminism. From State-Managed Capitalism to Neoliberal Crisis. Londres: Verso.

- (2017). "Crisis of Care? On the Social-Reproductive Contradictions of Contemporary Capitalism", en BHATTACHARYA, Tithi (ed.) (2017). Social Reproduction Theory. Remapping Class, Recentering Oppression. Londres: Pluto Press.

GAGO, Verónica (2015). La razón neoliberal. Economías barrocas y pragmática popular. Madrid: Traficantes de Sueños.

LÓPEZ ÁlVAREZ, Pablo (2010). "Biopolítica, liberalismo y neoliberalismo: acción política y gestión de la vida en el último Foucault", en ARRIBAS, Sonia; CANO, Germán; UGARTE, Javier (coords.) (2010). Hacer vivir, dejar morir. Biopolítica y capitalismo. Madrid: CSIC/La Catarata.

MARSHALL, T.H.; BOTTOMORE, Tom (2007). Ciudadanía y clase social. Madrid: Alianza Editorial.

MARX, Karl (2007). Salario, precio y ganancia. Madrid: La Lucerna.

— (2017) El Capital. Libro primero. Madrid: Siglo XXI.

MORINI, Cristina (2014). Por amor o a la fuerza. Feminización del trabajo y biopolítica del cuerpo. Madrid: Traficantes de Sueños.

MUÑOZ LEÓN, Fernando (2013). "Ciudadanía laboral: crítica y defensa de un concepto jurídico-político". Revista de Derecho Universidad Católica del Norte, Año 20 (2), pp.373-404.

PECK, Jamie (2003) "The rise of the workfare state". Kurswechsel: Zeitschrift für gessellschafts, wirtschafts und umweltpolitische Alternativen, 3, pp. 75-87.

PÉREZ OROZCO, Amaia (2017). Subversión feminista de la economía. Aportes para un debate sobre el conflicto capital-vida. Madrid: Traficantes de Sueños. 
SANTOS, Boaventura de Sousa (2011). El milenio huérfano. Ensayos para una nueva cultura política. Madrid: Trotta.

VÁZQUEZ GARCÍA, Francisco. (2017). “Del welfare al wellness: las tecnologías del bienestar y el gobierno de las subjetividades en el liberalismo avanzado" [Ponencia presentada en Primer Encuentro Interdisciplinar sobre el Bienestar (Cádiz, 18-20 abril 2017). Universidad de Cádiz]. Consultado el 19 de octubre de 2018, Academia.edu, en <https://www.academia.edu/36387067/_Del_Welfare_al_Wellness_las_tecnolog\%C3\%ADas_del_bienestar_y_el_gobierno_de_las_subjetividad es en el liberalismo avanzado en Primer Encuentro Interdisciplinar sobre el Bienestar_Cádiz 18-20 abril 2017 Universidad de Cádiz>.

Leyes citadas

Constitución de la República Española (9 diciembre 1931). Consultado el 5 septiembre de 2018, en <http://www.congreso.es/docu/constituciones/1931/1931_cd.pdf>.

Les accords de Matignon (7 junio 1936). Consultado el 6 septiembre de 2018, en $<$ https://travail-emploi.gouv.fr/IMG/pdf/Les accords de Matignon7juin36.pdf>.

National Industry Recovery Act [NIRA] (16 junio 1933). Consultado el 5 septiembre de 2018, en <https://www.ourdocuments.gov/doc.php?flash=false\&doc=66\&page=transcript $>$. 